\title{
Fast Power Density Assessment of 5G Mobile Handset Using Equivalent Currents Method
}

\author{
Wang He, Bo Xu, Member, IEEE, Lucia Scialacqua, Zhinong Ying, Senior Member, IEEE, \\ Alessandro Scannavini, Lars Jacob Foged, Senior Member, IEEE, Kun Zhao, \\ Carla Di Paola, Student Member, IEEE, Shuai Zhang, Senior Member, IEEE, Sailing He, Fellow, IEEE
}

\begin{abstract}
As the fifth-generation (5G) mobile communication is utilizing millimeter-wave (mmWave) frequency bands, electromagnetic field (EMF) exposure emitted from a 5G mmWave mobile handset should be evaluated and compliant with the relevant EMF exposure limits in terms of peak spatial-average incident power density. In this work, a fast power density (PD) assessment method for a $5 \mathrm{G}$ mmWave mobile handset using the equivalent currents (EQC) method is proposed. The EQC method utilizes the intermediate-field (IF) data collected by a spherical measurement system to reconstruct the EQCs over a reconstruction surface, and then computes the $P D$ in close proximity of the mobile handset with acceptable accuracy. The performance of the proposed method is evaluated using a mmWave mobile handset mock-up equipped with four quasiYagi antennas. The assessed PD results are compared with those computed using full-wave simulations and also those measured with a planar near-field (NF) scanning system. In addition, three influencing factors related to the accuracy of the EQC method, namely, the angular resolution, the phase error, and the handset position in the IF measurements, are also analyzed. The proposed method is a good candidate for fast PD assessment of EMF exposure compliance testing in the mmWave frequency range.
\end{abstract}

Index Terms-5G, antenna measurement, EMF exposure, equivalent currents, millimeter-wave, power density, source reconstruction.

\section{INTRODUCTION}

$\mathbf{T}$ HE fifth-generation (5G) communication technology is coming into commercial use. The millimeterwave (mmWave) frequency bands ranging from $24.25 \mathrm{GHz}$ to $29.5 \mathrm{GHz}$ (including n257, n258, and n261) and from $37 \mathrm{GHz}$ to $40 \mathrm{GHz}$ (n260), also known as the frequency range 2 (FR2),

The work is partially supported by National Key Research and Development Program of China (No. 2018YFC1407500) and the National Natural Science Foundation of China (11621101).

W. He and S. He are with the Centre for Optical and Electromagnetic Research, College of Optical Science and Engineering, Zhejiang University, Hangzhou 310058, China. S. He is also with the Department of Electromagnetic Engineering, KTH Royal Institute of Technology, SE-100 44 Stockholm, Sweden (e-mail: sailing@kth.se).

B. $\mathrm{Xu}$ is with Ericsson Research, Ericsson AB, SE-164 80 Stockholm, Sweden, and also with the Department of Electromagnetic Engineering, KTH Royal Institute of Technology, SE-100 44 Stockholm, Sweden.

L. Scialacqua, A. Scannavini, and L. J. Foged are with the Microwave Vision Group, Pomezia 00071, Italy.

Z. Ying and K. Zhao are with the Sony Research Center, Sony Corp., SE22188 Lund, Sweden. K. Zhao is also with the Antenna, Propagation and mm-Wave Section (APMS) at the Department of Electronic Systems, Aalborg University, 9220 Aalborg, Denmark.

C. D. Paola and S. Zhang are with the Antenna, Propagation and mm-Wave Section (APMS) at the Department of Electronic Systems, Aalborg University, 9220 Aalborg, Denmark. have been allocated to 5G New Radio (NR) [1]. Same as the previous generations of mobile communication technologies, radio frequency (RF) electromagnetic field (EMF) exposure from a $5 \mathrm{G}$ mmWave mobile handset is required to comply with the relevant exposure limits [2]-[5]. According to the draft international RF EMF exposure guidelines published by the International Commission on Non-Ionizing Radiation Protection (ICNIRP) [2] and the recently updated IEEE standard [3], above $6 \mathrm{GHz}$ the RF EMF exposure may be evaluated in terms of peak spatial-average incident power density ( $p s a P D)$ in free space (reference levels). There are very few studies on the new power density metric above $6 \mathrm{GHz}$, i.e., the socalled transmitted power density or epithelial power density, which is the power density in tissue (basic restriction). For brevity, we refer power density (PD) to incident power density in this paper. The $p s a P D$ is averaged over either $1 \mathrm{~cm}^{2}$ or $4 \mathrm{~cm}^{2}$ depending on the requirements of the relevant exposure guidelines [2]-[5]. A brief summary of the recent update of the RF EMF exposure guidelines above $6 \mathrm{GHz}$ can be found in [6].

Up to quite recently, not much attention has been paid to evaluating PD in close proximity of the mobile handset, due to a lack of applications in mmWave frequencies. With the emergence of $5 \mathrm{G}$, research around the rationale of the EMF exposure limits above $6 \mathrm{GHz}$ has been carried out (e.g. [7]-[18]), and new methodologies to measure the PD have been developed during the past few years [19]. Using the magnitude of the electric field, it is shown in [20] and [21] that PD assessment based on the conventional magnitude-based field combination will lead to very conservative $p s a P D$ for 5G mmWave mobile handset, while accurate PD assessment needs to involve both the magnitude and phase of the electric and magnetic fields. Using probes based on the pseudo-vector sensor design, a PD measurement method to reconstruct PD with the information of electric-field polarization ellipse is presented in [22]. This technique is used in the first commercial PD measurement system [23]. A two-probe method is applied in [24] to evaluate PD by directly measuring the electric and the magnetic fields. In [25] and [26], the electric field at a relatively far distance is measured with the traditional planar near-field (NF) antenna measurement system, and then plane wave spectrum expansion is applied to compute PD at a closer distance. Based on the solution of an inverse source problem, a PD measurement method was developed in [27] by measuring the amplitude and phase of the electric field, and a novel calibration technique was introduced when measuring 


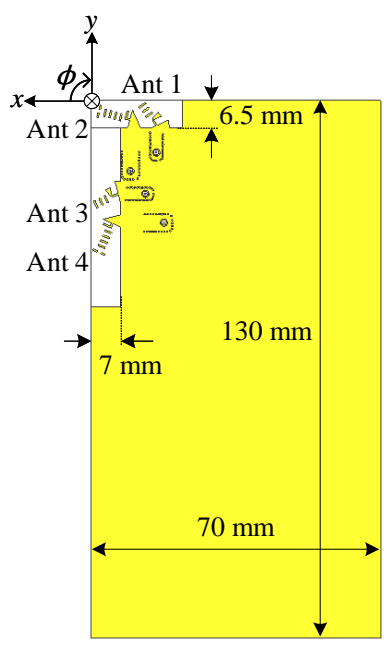

(a)

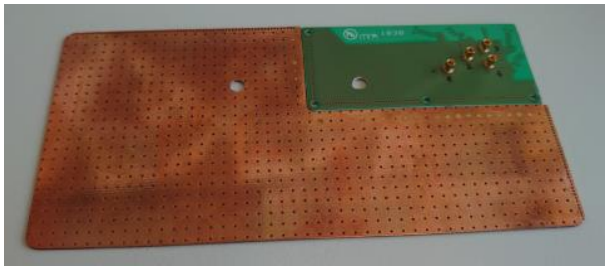

(c)

Fig. 1. (a) The bottom view and (b) the top view of the $5 \mathrm{G}$ mobile handse mock-up model equipped with four quasi-Yagi antennas and (c) the fabricated prototype.

very close to the device under test (DUT). However, planar scanning systems are employed in all these works, and a typical measurement of the fields on a limited planar area for each beam realization or each antenna port requires many hours. One can only assess the PD at one evaluation distance per measurement, or only obtain results at one frequency per measurement. In addition, considering a user equipment like a cuboid shaped mobile handset, these measurement methods can only evaluate PD on one side of the mobile handset per measurement. Repetitive measurements are needed on every side of the mobile handset to obtain $p s a P D$ in the entire space. In practice, PD compliance testing for one $5 \mathrm{G}$ mmWave product with up to dozens or even hundreds of beam realizations (see e.g., [28] and [29]) can take days or even weeks due to the aforementioned reasons. Therefore, a timeefficient PD assessment technology is urgently needed while $5 \mathrm{G}$ mmWave is being extensively deployed.

For SAR compliance testing below $6 \mathrm{GHz}$, fast SAR evaluation techniques [30] are allowed with the purpose of identifying the highest SAR conditions or relatively high SAR conditions. Only under these identified SAR conditions, the full SAR testing are performed, and thus the total time of compliance testing can be significantly reduced. In this paper, we apply a fast PD assessment with a similar logic, i.e., a fast equivalent currents (EQC) method [31]-[41] is used to identify the direction in which the maximum PD appears, and then further related measurements and analyses are performed in this direction. Based on the dual-equation formulation [39]-

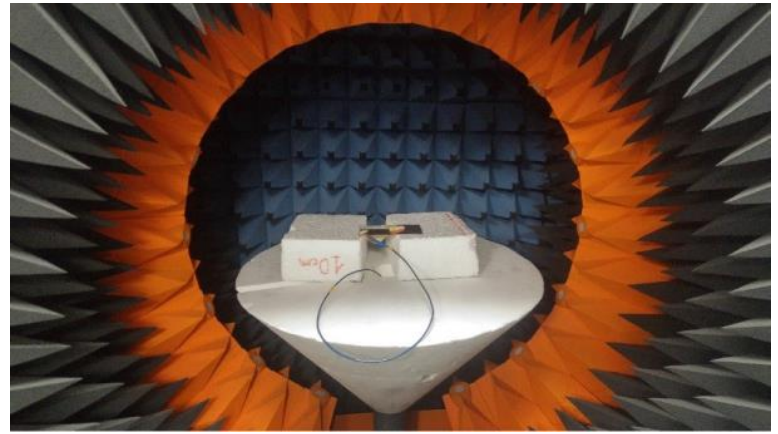

(a)

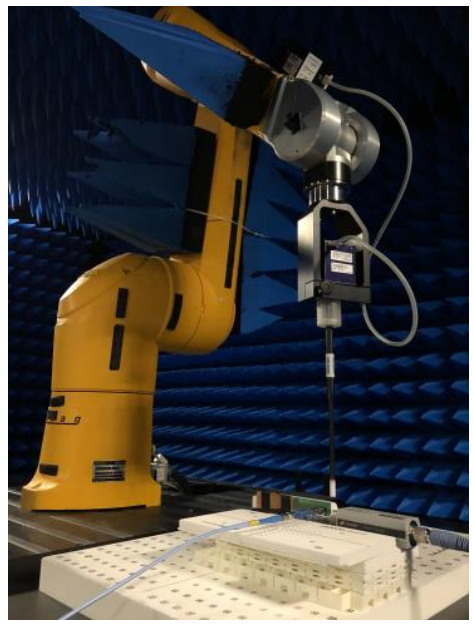

(b)

Fig. 2. (a) The measured IF data is collected by MVG StarLab $50 \mathrm{GHz}$ and (b) the NF is measured by SPEAG cDASY6 mmWave Module V1.0 and EUmmWV3 probe.

[41] of the EQC method, the PD assessment is conducted on a $5 \mathrm{G}$ mmWave mobile handset mock-up (DUT in this work) equipped with four quasi-Yagi antennas. The electric field of the DUT is measured by MVG StarLab $50 \mathrm{GHz}$ [42] in the intermediate-field (IF) region, i.e., the transition region between the NF and the far-field (FF). Using the measured IF data incorporated into the EQC method, the PD distribution in close proximity of the DUT is reconstructed, and psaPD is calculated at different evaluation distances from the DUT. The results are compared with those calculated with the simulated IF data incorporated into the EQC method, as well as those computed from the direct full-wave simulations and those from the reference PD measurements. Some factors influencing the accuracy of the PD assessed with the EQC method are analyzed, including the angular resolution, the phase error, and the DUT position in the IF measurements.

\section{MOdEL AND Methods}

\section{A. 5G Mobile Handset Mock-up}

Fig. 1 shows the $5 \mathrm{G}$ mmWave mobile handset mock-up [43] employed in this work. The size of the handset mockup model is $70 \mathrm{~mm} \times 130 \mathrm{~mm} \times 0.76 \mathrm{~mm}$. The substrate is Rogers RO3003 with permittivity of 3 and loss tangent of 0.001 . Without loss of generality, a beam switch antenna array composed of four quasi-Yagi antennas are used for PD 

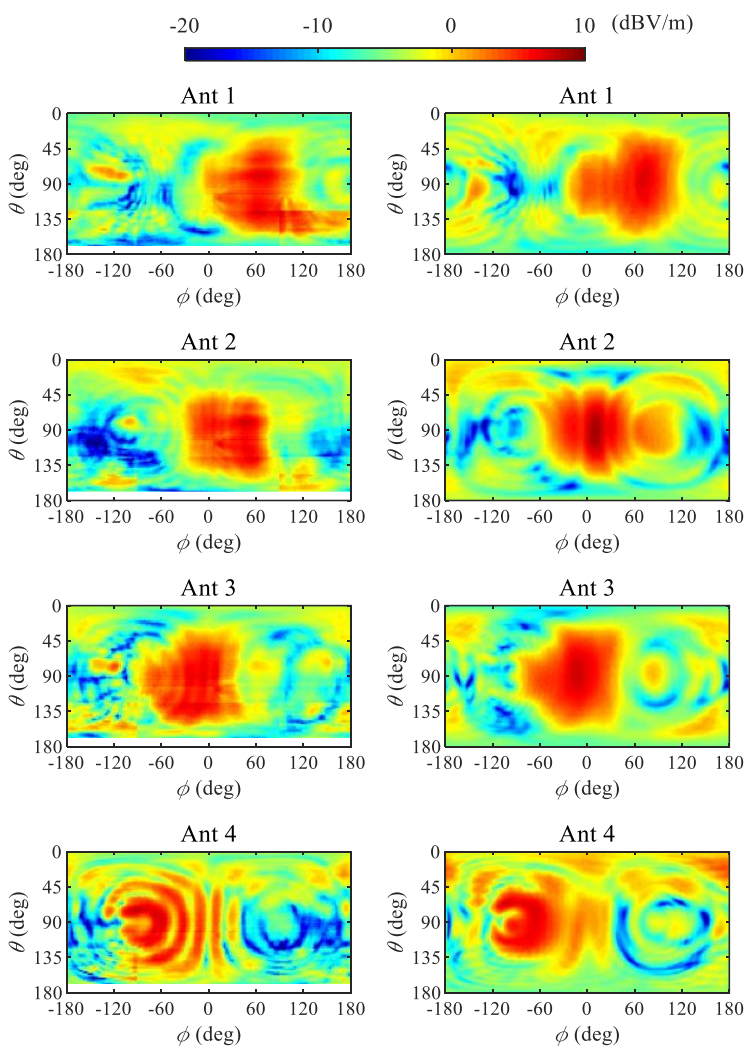

(a) Measured

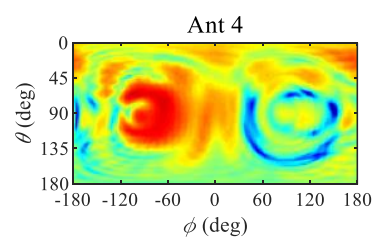

(b) Simulated

Fig. 3. (a) The measured and (b) simulated $|\boldsymbol{E}|$ distributions over $\Sigma_{\mathrm{M}}$, a sphere with radius of $450 \mathrm{~mm}$, in the IF region of Ant $1-$ Ant 4 at $28 \mathrm{GHz}$. The input power is normalized to $5 \mathrm{dBm}$.

assessment. The four quasi-Yagi antennas are printed in the top-right corner of the substrate, denoted in the figures as Ant 1-Ant 4. The antennas are connected to microstrip lines fed by the SMPS connectors [44]. In order to cover the right hemisphere space (see Fig. 1(b)), the four quasi-Yagi antennas are bent toward different directions. In this work, the quasiYagi antennas are excited at $28 \mathrm{GHz}$ and $38 \mathrm{GHz}$. More details about the antenna design can be found in [43]. The PD results for each antenna are normalized to $5 \mathrm{dBm}$ input power in both simulations and measurements.

\section{B. Equivalent Currents Method and Intermediate-Field Mea- surements}

In the EQC method, the EQCs are reconstructed over a closed reconstruction surface $\Sigma_{\mathrm{R}}$ of arbitrary shape using the measured or simulated field data, and then the EQCs are treated as the sources for the radiated electric field and magnetic field outside $\Sigma_{\mathrm{R}}$. The EQC method can be divided into single-equation formulation and dual-equation formulation. Single-equation formulation [35]-[37] has only integral equations and cannot give good accuracy of the EQCs under the definition of Love's equivalence, while dual-equation formulation has not only integral equations but also boundary integral identities to enforce the reconstructed EQCs over $\Sigma_{R}$ to be in Love's form [39]-[41].
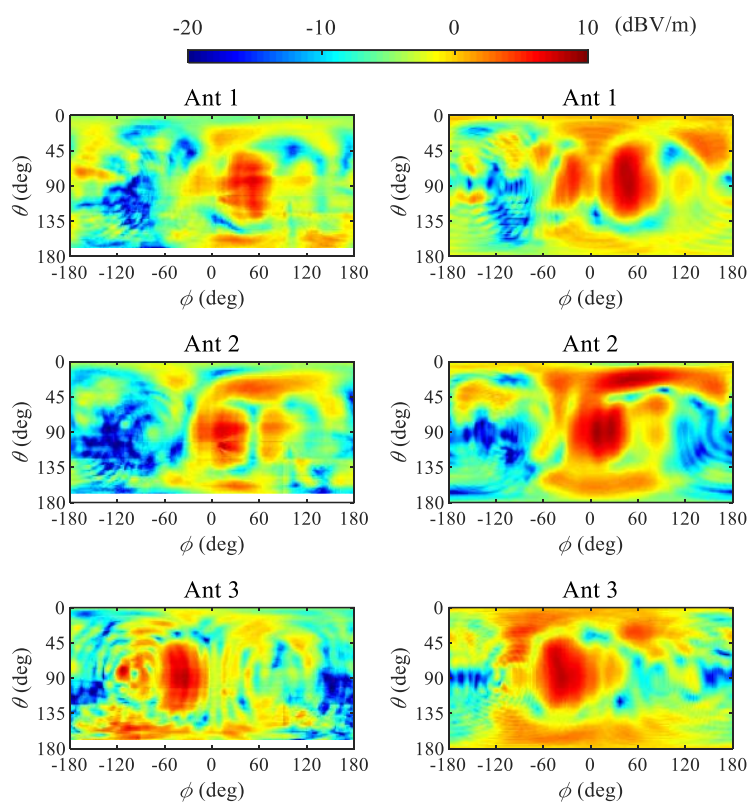

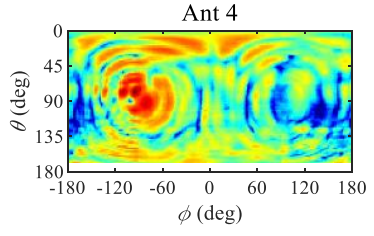

(a) Measured

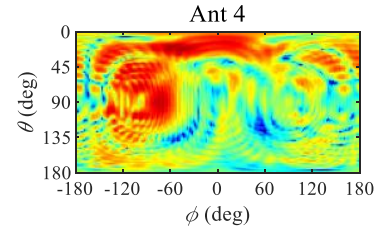

(b) Simulated
Fig. 4. (a) The measured and (b) simulated $|\boldsymbol{E}|$ distributions over $\Sigma_{\mathrm{M}}$, a sphere with radius of $450 \mathrm{~mm}$, in the IF region of Ant $1-$ Ant 4 at $38 \mathrm{GHz}$. The input power is normalized to $5 \mathrm{dBm}$.

The EQC method based on dual-equation formulation, implemented in MVG INSIGHT [45], is performed in this work. The EQCs are reconstructed over $\Sigma_{R}$, which in this work is a cuboid enclosing the DUT with a gap of $3 \mathrm{~mm}$. The integral equation is established by taking the enforcement on the EQCs, i.e., $\boldsymbol{J}_{\text {eq }}$ and $\boldsymbol{M}_{\text {eq }}$, radiating the same tangential components as the IF over measurement surface $\Sigma_{M}$ [39]-[41]:

$$
\hat{\boldsymbol{n}} \times \boldsymbol{E}(\boldsymbol{r})=\hat{\boldsymbol{n}} \times\left[-\eta_{0} \mathcal{L}\left(\boldsymbol{J}_{\mathrm{eq}} ; \boldsymbol{r}\right)+\mathcal{K}\left(\boldsymbol{M}_{\mathrm{eq}} ; \boldsymbol{r}\right)\right], \quad \boldsymbol{r} \in \Sigma_{\mathrm{M}}
$$

where

$$
\begin{aligned}
\mathcal{L}\left(\boldsymbol{J}_{\mathrm{eq}} ; \boldsymbol{r}\right) & =\mathrm{j} k_{0} \int_{\Sigma_{\mathrm{R}}}\left[\boldsymbol{J}_{\mathrm{eq}}\left(\boldsymbol{r}^{\prime}\right)+\frac{1}{k_{0}^{2}} \nabla \nabla_{s}^{\prime} \cdot \boldsymbol{J}_{\mathrm{eq}}\left(\boldsymbol{r}^{\prime}\right)\right] g\left(\boldsymbol{r}, \boldsymbol{r}^{\prime}\right) \mathrm{d} s^{\prime} \\
\mathcal{K}\left(\boldsymbol{M}_{\mathrm{eq}} ; \boldsymbol{r}\right) & =\int_{\Sigma_{\mathrm{R}}} \boldsymbol{M}_{\mathrm{eq}}\left(\boldsymbol{r}^{\prime}\right) \times \nabla g\left(\boldsymbol{r}, \boldsymbol{r}^{\prime}\right) \mathrm{d} s^{\prime} \\
g\left(\boldsymbol{r}, \boldsymbol{r}^{\prime}\right) & =\frac{e^{-\mathrm{j} k_{0}\left|\boldsymbol{r}-\boldsymbol{r}^{\prime}\right|}}{4 \pi\left|\boldsymbol{r}-\boldsymbol{r}^{\prime}\right|}
\end{aligned}
$$

where $\eta_{0}=\sqrt{\mu_{0} / \epsilon_{0}}, k_{0}=\omega \sqrt{\mu_{0} \epsilon_{0}}$, and $\nabla_{s}^{\prime}$ is the surface divergence operator. The Love's form of EQCs is enforced by the following boundary integral identities:

$$
\begin{aligned}
\hat{\boldsymbol{n}} \times\left[-\eta_{0} \mathcal{L}\left(\boldsymbol{J}_{\mathrm{eq}} ; \boldsymbol{r}\right)+\mathcal{K}\left(\boldsymbol{M}_{\mathrm{eq}} ; \boldsymbol{r}\right)\right] & =-\frac{1}{2} \boldsymbol{M}_{\mathrm{eq}}(\boldsymbol{r}), \boldsymbol{r} \in \Sigma_{\mathrm{R}}, \\
\hat{\boldsymbol{n}} \times\left[-\frac{1}{\eta_{0}} \mathcal{L}\left(\boldsymbol{M}_{\mathrm{eq}} ; \boldsymbol{r}\right)-\mathcal{K}\left(\boldsymbol{J}_{\mathrm{eq}} ; \boldsymbol{r}\right)\right] & =\frac{1}{2} \boldsymbol{J}_{\mathrm{eq}}(\boldsymbol{r}), \boldsymbol{r} \in \Sigma_{\mathrm{R}} .(3)
\end{aligned}
$$




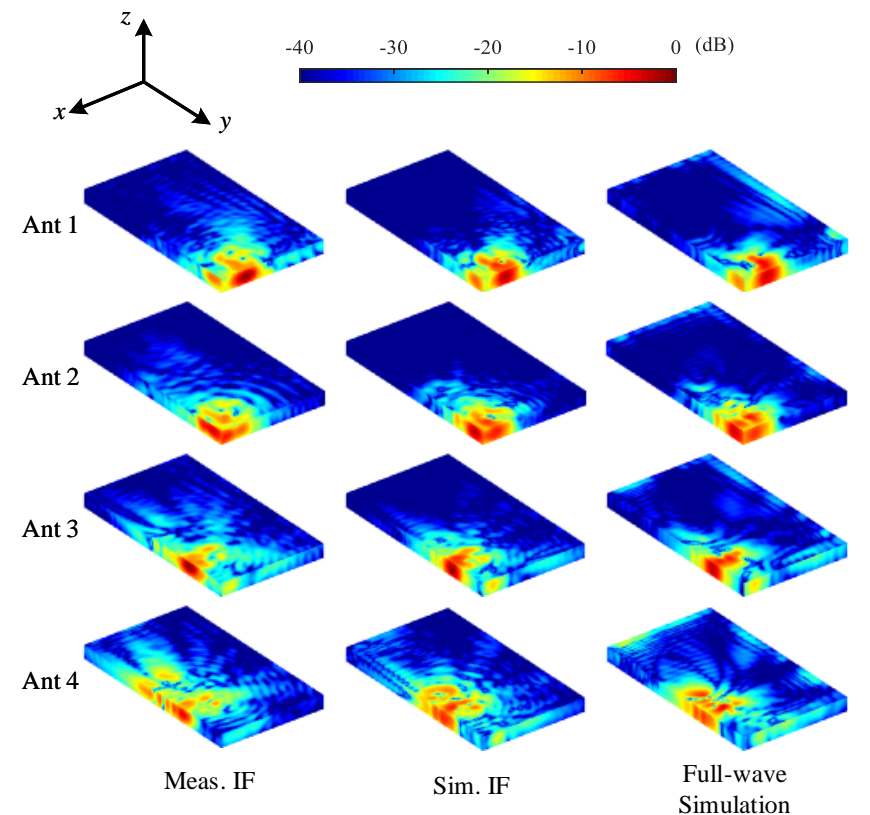

Fig. 5. The PD distributions at $28 \mathrm{GHz}$ reconstructed from measured IF (left column), reconstructed from simulated IF (middle column) and computed from full-wave simulation (right column) on the outer surface of a closed box embracing the DUT from the outside with a $5 \mathrm{~mm}$ gap. The PD distributions are normalized with $20 \mathrm{~W} / \mathrm{m}^{2}$.

The system of equations (1)-(3) is solved with the standard method of moment (MoM), employing discretization of $\Sigma_{R}$ in terms of the Rao-Wilton-Glisson (RWG) basis [46].

The measurement surface $\Sigma_{\mathrm{M}}$ in this work is spherical with a radius of $450 \mathrm{~mm}$, over which the measured IF data is obtained using the spherical measurement system StarLab $50 \mathrm{GHz}$, as shown in Fig. 2(a). The system consists of the probes embedded in a rotating arch able to scan the elevation angles, and a round table to rotate a DUT on the azimuth plane. Probes are covered by absorbing material in the wedge shape while the round table is made of foam to reduce its impact on DUT radiation. During the measurements, each antenna under test is placed on the round table with the center of the SMPS connector positioned at the origin of the spherical coordinate system. The IF data on the full sphere are collected by electronically scanning the probe array in elevation and rotating the DUT in azimuth. Due to the fact the probe array structure can rotate mechanically around its center, the angular resolution of the IF data can be selected based on the DUT size and frequency to be tested. Specifically, the angular resolution of the IF data is set to $\Delta \alpha=2^{\circ}$ for these measurements. The measured $|\boldsymbol{E}|$ distributions over $\Sigma_{\mathrm{M}}$ are shown in Fig. 3(a) and Fig. 4(a) for $28 \mathrm{GHz}$ and $38 \mathrm{GHz}$, respectively. The simulated IF data is also computed with the similar settings as in the measurements, and its $|\boldsymbol{E}|$ distributions are shown in Fig. 3(b) and Fig. 4(b). The electric field and the magnetic field outside $\Sigma_{\mathrm{R}}$ are computed in the full-wave simulation software CST Studio Suite [47], using the obtained measurement-based and simulation-based EQCs for comparison. For the IF measurements, the measurement time for each antenna only takes about 10 minutes for a wide

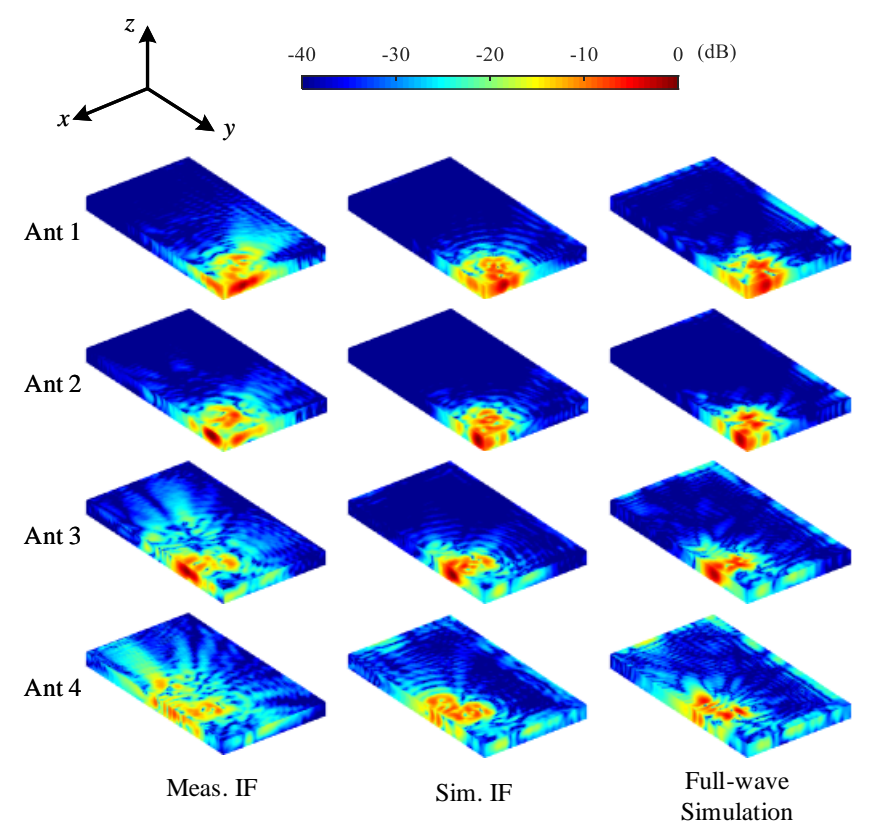

Fig. 6. The PD distributions at $38 \mathrm{GHz}$ reconstructed from measured IF (left column), reconstructed from simulated IF (middle column) and computed from full-wave simulation (right column) on the outer surface of a closed box embracing the DUT from the outside with a $5 \mathrm{~mm}$ gap. The PD distributions are normalized with $20 \mathrm{~W} / \mathrm{m}^{2}$.

band including $28 \mathrm{GHz}$ and $38 \mathrm{GHz}$.

\section{Reference Direct Simulation and Power Density Measure- ments}

As a reference, the electric field and the magnetic field in close proximity of the DUT are computed in CST. In addition, the direct $\mathrm{NF}$ measurements are performed at $28 \mathrm{GHz}$ for Ant 2, Ant 3, and Ant 4 in the $+x$-direction (direction of the maximum PD, as demonstrated in section III) at the evaluation distance $\mathrm{d}$ of $5 \mathrm{~mm}, 10 \mathrm{~mm}, 15 \mathrm{~mm}, 20 \mathrm{~mm}$ and $25 \mathrm{~mm}$ using SPEAG cDASY6 mmWave Module V1.0 [23] and EUmmWV3 probe [48] (see Fig. 2(b)). To save experiment time, we did not measure Ant 1, whose maximum PD is in the $+y$-direction. The scanning step is a quarter of freespace wavelength. The expanded uncertainty is $2.2 \mathrm{~dB}$ for the reference NF measurements [23]. Depending on the size of the scan area, one or a few hours are required to carry out the reference NF measurement for one antenna, at one frequency point, and at one distance.

\section{Spatial-Average Power Density}

The spatial-average PD, i.e., the power flux density averaged over an area $A$, can be written as [19]:

$$
S=\frac{1}{2 A} \int_{A} \operatorname{Re}\left[\boldsymbol{E} \times \boldsymbol{H}^{*}\right] \cdot \hat{\boldsymbol{n}} \mathrm{d} A,
$$

where $\hat{\boldsymbol{n}}$ is the unit vector normal to $A$. The point PD can be computed by taking $A \rightarrow 0$. In this paper, the averaging area $A$ is of square shape. At each evaluation distance $\mathrm{d}$ normal to the respective surface of the DUT, the averaging area is scanned over the plane parallel to that surface of DUT. For 

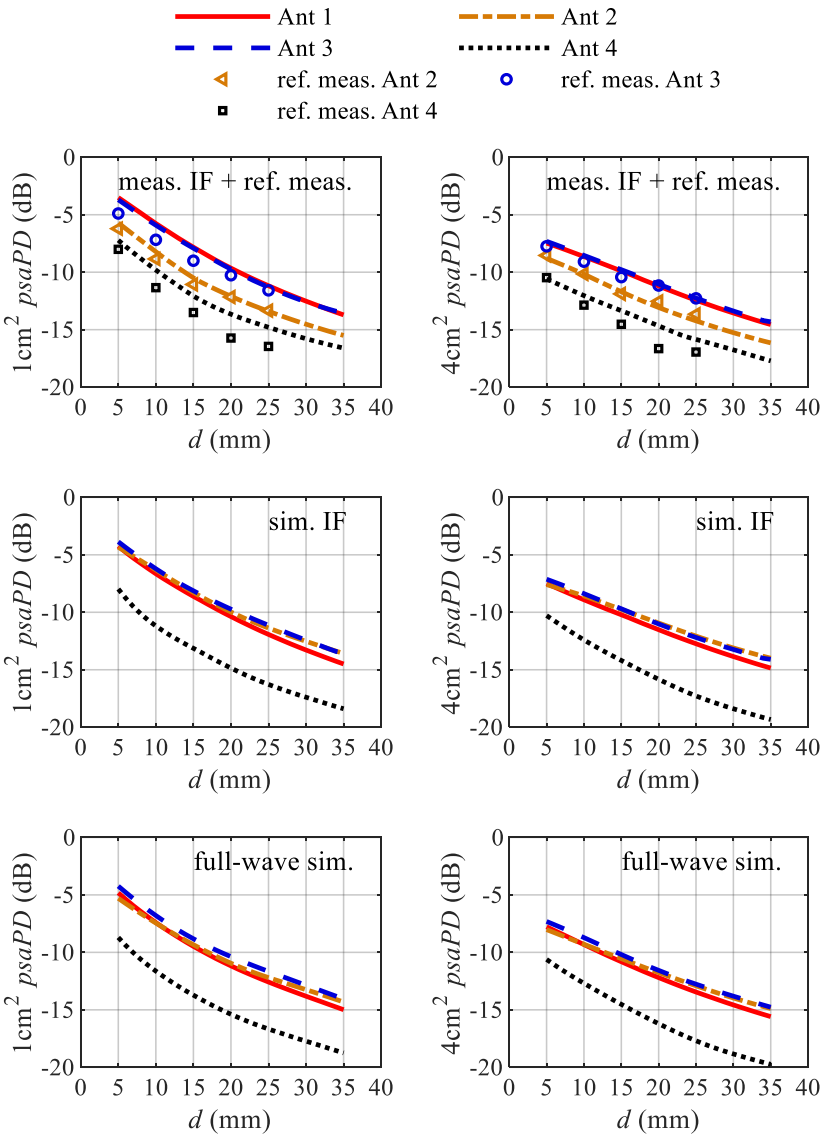

Fig. 7. The $1 \mathrm{~cm}^{2}$ (left column) and $4 \mathrm{~cm}^{2}$ (right column) psaPD at $28 \mathrm{GHz}$ as the evaluation distance varies for Ant 1-Ant 4 . The values are normalized with $20 \mathrm{~W} / \mathrm{m}^{2}$.

TABLE I

POINT PD RECONSTRUCTION ERROR OF ANT 1-ANT 4 EVALUATED AT $d=5 \mathrm{~mm}$ USING SAMPLES FROM A $10 \times 10$ GRID WITH $2 \mathrm{~mm}$ STEP (UNIT: dB).

\begin{tabular}{ccccc}
\hline \hline & Ant 1 & Ant 2 & Ant 3 & Ant 4 \\
\hline $28 \mathrm{GHz}$ & 0.8 & 0.7 & 0.5 & 1.1 \\
\hline $38 \mathrm{GHz}$ & 0.8 & 0.9 & 0.9 & 0.9 \\
\hline \hline
\end{tabular}

a user equipment like a cuboid shaped mobile handset, PD in six directions, i.e. $\pm x, \pm y$, and $\pm z$, normal to respective surfaces needs to be evaluated for compliance testing. The general expression of $p s a P D$ at $d$ is:

$$
\operatorname{psa} P D(d)=\max _{\text {all areas at } d}\left\{\frac{1}{2 A} \int_{A} \operatorname{Re}\left[\boldsymbol{E} \times \boldsymbol{H}^{*}\right] \cdot \hat{\boldsymbol{n}} \mathrm{d} A\right\} .
$$

For the sake of brevity, psaPD averaged over $1 \mathrm{~cm}^{2}$ and $4 \mathrm{~cm}^{2}$ are denoted by $1 \mathrm{~cm}^{2}$ psaPD and $4 \mathrm{~cm}^{2} p s a P D$, respectively.

\section{Power Density Assessment Results}

Fig. 5 and Fig. 6 show the point PD distributions on the outer surface of the closed box that embraces the DUT with a $5 \mathrm{~mm}$ gap on each side at $28 \mathrm{GHz}$ and $38 \mathrm{GHz}$, respectively. $5 \mathrm{~mm}$ is selected according to the European Committee for Electrotechnical Standardization (CENELEC) standard EN 50566 [49] for EMF compliance assessments of body-worn
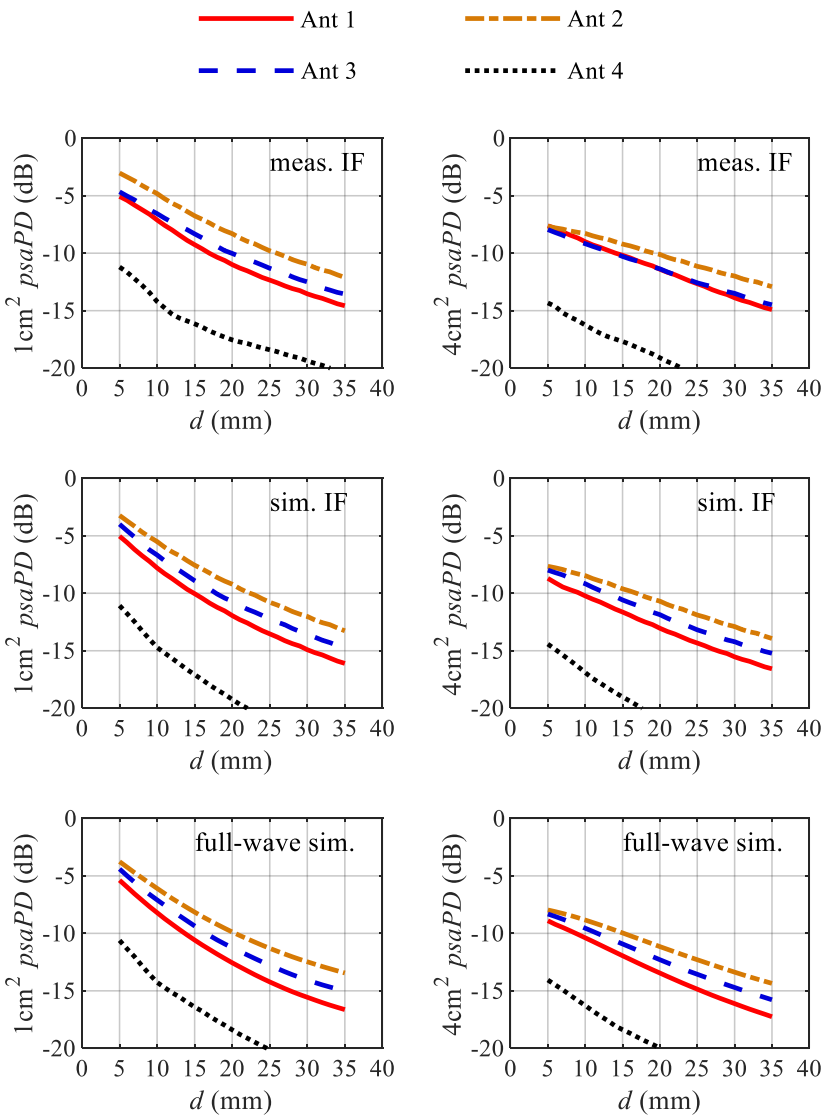

Fig. 8. The $1 \mathrm{~cm}^{2}$ (left column) and $4 \mathrm{~cm}^{2}$ (right column) psaPD at $38 \mathrm{GHz}$ as the evaluation distance varies for Ant 1-Ant 4. The values are normalized with $20 \mathrm{~W} / \mathrm{m}^{2}$.

devices. Though the European standard [49] is applied only up to $6 \mathrm{GHz}$ for SAR measurements, the same criteria is chosen here due to the lack of available standards on PD measurements. The PD distributions are reconstructed using the measured IF data and the simulated IF data. The distributions obtained through the direct full-wave simulations are also presented. The figures highlight that the point PD distributions from the measured IF and the simulated IF are similar to those from the direct full-wave simulations in general. Some deviation between measurement-based results and simulationbased results may be attributed to some errors in fabrication and the test platform used in the IF measurements. Moreover, the figures show that the maximum PD is in the $+y$-direction for Ant 1 and in the $+x$-direction for Ant 2, Ant 3, and Ant 4 . Further analyses are given only in these directions for the respective antennas.

Similar to the simulation uncertainty of SAR below $6 \mathrm{GHz}$ [50], the uncertainty of the EQC method in the PD assessment can be quantified as a relative difference between the point PD reconstructed from the simulated IF, $S^{\text {rec }}$, and the point PD from the direct full-wave simulations, $S^{\text {ref }}$,

$$
U=\frac{\max \left[\left|S^{\mathrm{rec}}(\boldsymbol{r})-S^{\mathrm{ref}}(\boldsymbol{r})\right|\right]}{\max \left[S^{\mathrm{ref}}(\boldsymbol{r})\right]} .
$$

In the context of PD assessment, we call $U$ the point PD 
reconstruction error hereafter. For each antenna, the samples from a $10 \times 10$ grid with $2 \mathrm{~mm}$ step at $d=5 \mathrm{~mm}$ in the maximum PD direction are taken to calculate $U$. Table I summarizes the $U$ values in $\mathrm{dB}$ for Ant $1-$ Ant 4 at both $28 \mathrm{GHz}$ and $38 \mathrm{GHz}$. The maximum $U$ for all the investigated antennas and frequencies is $1.1 \mathrm{~dB}$.

Fig. 7 and Fig. 8 show the $1 \mathrm{~cm}^{2}$ psaPD and $4 \mathrm{~cm}^{2}$ psaPD for Ant $1-$ Ant 4 , at $28 \mathrm{GHz}$ and $38 \mathrm{GHz}$, respectively. The levels of $p s a P D$ reconstructed from the simulated IF data agree well with the psaPD levels from the corresponding full-wave simulations. At $28 \mathrm{GHz}$, the levels of psaPD reconstructed from the measured IF also agree well with those obtained from the reference NF measurements considering the uncertainty of the measurement systems. As mentioned before, the difference between the measurement-based and simulation-based results may be due to some errors in fabrication and the test environments. As can be seen from the figures, both the measurementbased results and simulation-based results indicate that the EQC method can be used to identify the beams with the relatively high psaPD levels.

\section{Analysis of Measurement Requirements}

StarLab $50 \mathrm{GHz}$ is designed for passive and active (i.e., over-the-air, OTA) measurements of 5G NR terminals at the FR2 band [42]. For this effort, the OTA measurement system (StarLab $50 \mathrm{GHz}$ ) has been used for PD measurements. Specifically, this section will analyze the influence of angular resolution, phase error, and DUT position in the IF measurements. These three influencing factors are relevant to the present EQC method.

To describe the discrepancy between $p s a P D^{\text {rec }}$ (the psaPD reconstructed by the EQC method) and psaPD ${ }^{\text {ref }}$ (the reference $p s a P D$ directly computed from the full-wave simulation), the reconstruction error [22], [25] in $\mathrm{dB}$ can be written as:

$$
\operatorname{Error}(d)=\left|10 \log _{10}\left[\frac{p s a P D^{\mathrm{rec}}(d)}{\text { psaPD } D^{\mathrm{ref}}(d)}\right]\right| \text {. }
$$

In order to separate the error of the EQC method itself from other errors due to, e.g., the differences between the simulation and fabrication, only the simulation data is used to analyze the reconstruction error in this section.

\section{A. Intermediate-Field Angular Resolution}

The IF used for reconstruction is sampled with a certain angular resolution in the spherical coordinate system. Sparse sampling may result in poor accuracy, while dense sampling increases the measurement time in data collection and the reconstruction process, and also requires more complicated measurement equipment, e.g., denser probe implementation in the arc of spherical measurement systems. In this subsection, the reconstruction errors, corresponding to different angular resolutions, are investigated with the angular interval $\Delta \alpha$ of $2^{\circ}, 4^{\circ}, 6^{\circ}$, and $8^{\circ}$, in both the zenith and azimuthal angles.

The effects of angular resolution can be visualized through the $\left|\boldsymbol{J}_{\text {eq }}\right|$ distributions on $\Sigma_{\mathrm{R}}$, as can be seen in Fig. 9 for Ant 3 at $28 \mathrm{GHz}$ as an example. The distribution with $\Delta \alpha=$ $4^{\circ}$ is similar to the distribution with $\Delta \alpha=2^{\circ}$. For $\Delta \alpha=$

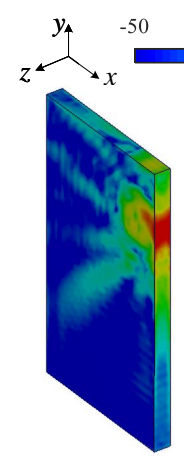

(a)

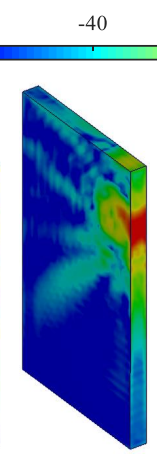

(b)

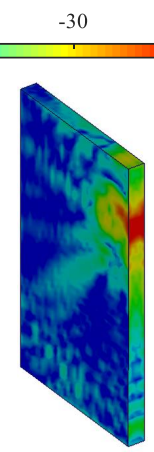

(c) $-20(\mathrm{dBA} / \mathrm{m})$

Fig. 9. $\left|\boldsymbol{J}_{\text {eq }}\right|$ distributions reconstructed from the simulated IF data of Ant 3 at $28 \mathrm{GHz}$ with the angular resolution of (a) $\Delta \alpha=2^{\circ}$, (b) $\Delta \alpha=4^{\circ}$, (c) $\Delta \alpha=6^{\circ}$, and (d) $\Delta \alpha=8^{\circ}$.

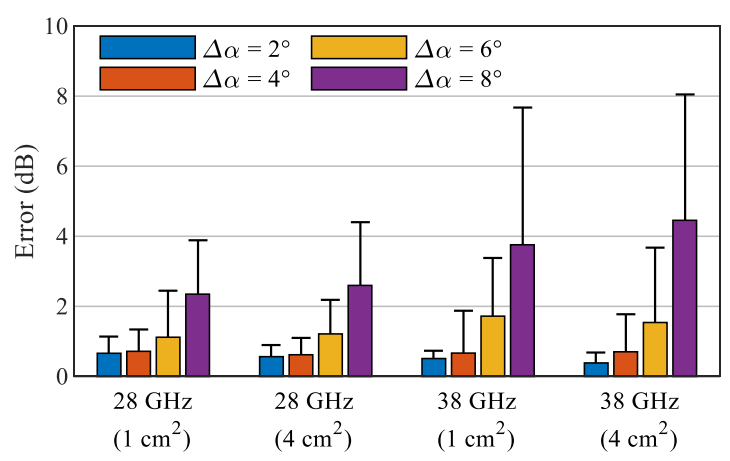

Fig. 10. The mean and maximum values of the reconstruction errors for the $1 \mathrm{~cm}^{2}$ and $4 \mathrm{~cm}^{2}$ psaPD at $28 \mathrm{GHz}$ and the $1 \mathrm{~cm}^{2}$ and $4 \mathrm{~cm}^{2}$ psaPD at $38 \mathrm{GHz}$ reconstructed from the simulated IF data with different values of angular resolution $\Delta \alpha$. Each bar contains 124 samples from different combinations of antenna (Ant 1-4) and evaluation distance (from $d=5 \mathrm{~mm}$ to $d=35 \mathrm{~mm}$ with $1 \mathrm{~mm}$ step).

$6^{\circ}$, more spread EQC appears, although the entire distribution still resembles that with $\Delta \alpha=2^{\circ}$ and $\Delta \alpha=4^{\circ}$. When $\Delta \alpha$ reaches $8^{\circ}$, the red region becomes smaller, the unexpected EQCs spread over wide areas, and the details in the $\left|\boldsymbol{J}_{\text {eq }}\right|$ distributions with $\Delta \alpha=2^{\circ}$ and $\Delta \alpha=4^{\circ}$ are almost lost. This can be explained with the degrees of freedom of the IF, which is the minimum number of samples necessary to realize the reconstruction [51]-[53]. For a spherical measurement system, the maximum $\Delta \alpha$ to meet the degrees of freedom of the field can be estimated by [40]

$$
(\Delta \alpha)_{\max }=\frac{1}{2 a / \lambda+10 / \pi},
$$

where $a$ is the radius of the minimum sphere enclosing the DUT. Using (8), $(\Delta \alpha)_{\max }$ for our DUT is about $3.4^{\circ}$ at $28 \mathrm{GHz}$ and about $2.6^{\circ}$ at $38 \mathrm{GHz}$. When $\Delta \alpha$ is larger than $(\Delta \alpha)_{\max }$, the reconstructed fields suffer from the information loss of the high-order spherical-wave modes.

Fig. 10 shows the reconstruction errors for different $\Delta \alpha$ for four cases, i.e., the $1 \mathrm{~cm}^{2}$ psaPD at $28 \mathrm{GHz}$, the $4 \mathrm{~cm}^{2}$ psaPD at $28 \mathrm{GHz}$, the $1 \mathrm{~cm}^{2}$ psaPD at $38 \mathrm{GHz}$, and the $4 \mathrm{~cm}^{2}$ psaPD at $38 \mathrm{GHz}$. In general, both the mean value and the maximum value of the errors gradually increase from $\Delta \alpha=2^{\circ}$ 


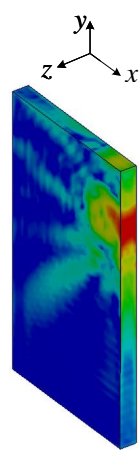

(a)

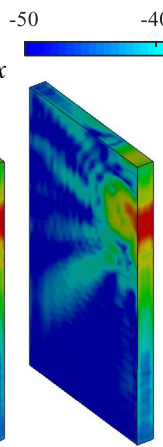

(b)

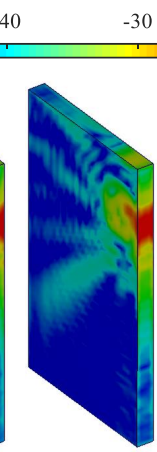

(c)

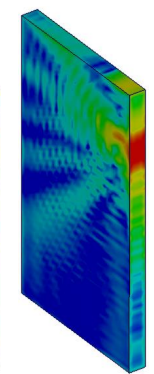

(d)
$-20(\mathrm{dBA} / \mathrm{m})$

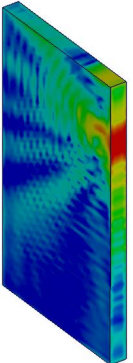

(e)
Fig. 11. $\left|\boldsymbol{J}_{\text {eq }}\right|$ distributions reconstructed from the simulated IF data of Ant 3 at $28 \mathrm{GHz}$ with the phase noise level of (a) $\sigma=0^{\circ}$, (b) $\sigma=5^{\circ}$, (c) $\sigma=10^{\circ}$, (d) $\sigma=15^{\circ}$, and (e) $\sigma=20^{\circ}$.

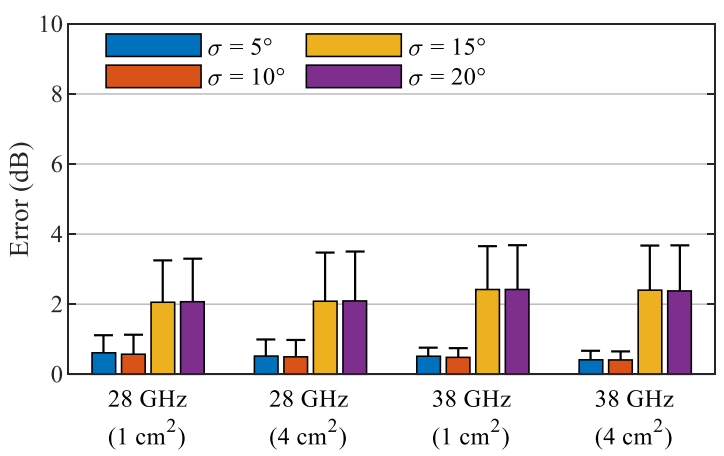

Fig. 12. The mean value and the $95 \%$ confidence range of the reconstruction errors for the $1 \mathrm{~cm}^{2}$ and $4 \mathrm{~cm}^{2}$ psaPD at $28 \mathrm{GHz}$ and the $1 \mathrm{~cm}^{2}$ and $4 \mathrm{~cm}^{2}$ psaPD at $38 \mathrm{GHz}$ reconstructed from the simulated IF data with different levels of noise. Each bar contains 620 samples from different combinations of $\phi_{\mathrm{n}}$ distribution (five distributions at each noise level), antenna (Ant 1-4), and evaluation distance (from $d=5 \mathrm{~mm}$ to $d=35 \mathrm{~mm}$ with $1 \mathrm{~mm}$ step).

TABLE II

Mean Value of Electric Field Data Misfit $\epsilon_{\mathrm{M}}$ For Ant 1-Ant 4 OVER $\Sigma_{\mathrm{M}}$ With DifFerent VALUES OF Phase Noise LEVEL $\sigma$ AT $28 \mathrm{GHz}$ AND $38 \mathrm{GHz}$

\begin{tabular}{cccccc}
\hline \hline$\sigma$ & $0^{\circ}$ & $5^{\circ}$ & $10^{\circ}$ & $15^{\circ}$ & $20^{\circ}$ \\
\hline $28 \mathrm{GHz}$ & 0.049 & 0.102 & 0.180 & 0.597 & 0.619 \\
\hline $38 \mathrm{GHz}$ & 0.076 & 0.121 & 0.193 & 0.621 & 0.648 \\
\hline
\end{tabular}

to $\Delta \alpha=8^{\circ}$. At $28 \mathrm{GHz}$, the mean and the maximum errors for $\Delta \alpha=4^{\circ}$ are almost the same as those for $\Delta \alpha=2^{\circ}$, while at $38 \mathrm{GHz}$, the maximum error for $\Delta \alpha=4^{\circ}$ is significantly larger than that for $\Delta \alpha=2^{\circ}$, implying that $\Delta \alpha=4^{\circ}$ is sufficient for $28 \mathrm{GHz}$ but insufficient for $38 \mathrm{GHz}$ in the PD assessment for a $5 \mathrm{G}$ mmWave mobile handset with a similar size. For a smaller DUT, a more relaxed requirement on $\Delta \alpha$ is possible.

\section{B. Phase Error of Intermediate-Field Measurement}

The phase measurement has greater uncertainty compared with the amplitude measurement, due to the noise in the measurement system [54]. To assess the effects of the phase error on the reconstructed $p s a P D$, at each angle $(\theta, \phi)$, phase noise $\phi_{\mathrm{n}}(\theta, \phi)$ is added to the phase of the simulated IF data $\boldsymbol{E}^{\mathrm{sim}}$ :

$$
\boldsymbol{E}_{\mathrm{n}}^{\operatorname{sim}}(\theta, \phi)=\boldsymbol{E}^{\operatorname{sim}}(\theta, \phi) e^{-\mathrm{j} \phi_{\mathrm{n}}(\theta, \phi)},
$$

where $\phi_{\mathrm{n}}(\theta, \phi) \sim N\left(0, \sigma^{2}\right) . \boldsymbol{E}_{\mathrm{n}}^{\mathrm{sim}}$ is used to reconstruct the corresponding EQCs and compute psaPD. The results reconstructed from $\boldsymbol{E}^{\mathrm{sim}}$, i.e., $\sigma=0^{\circ}$, are also computed for comparison.

Fig. 11 shows the $\left|\boldsymbol{J}_{\text {eq }}\right|$ distributions on $\Sigma_{\mathrm{R}}$ at $28 \mathrm{GHz}$ for Ant 3 with different phase noise level $\sigma$. The distributions for $\sigma=5^{\circ}$ and $\sigma=10^{\circ}$ are almost the same as that for $\sigma=0^{\circ}$, while the distributions for $\sigma=15^{\circ}$ and $\sigma=20^{\circ}$ contain strong ripples, indicating that a noise level up to $\sigma=10^{\circ}$ is acceptable. This can be further confirmed by computing the reconstruction errors (7) for different $\sigma$. In Fig. 12, at both $28 \mathrm{GHz}$ and $38 \mathrm{GHz}$, the mean value and the $95 \%$ confidence range of the reconstruction errors for $\sigma=5^{\circ}$ and $\sigma=10^{\circ}$ are small. When $\sigma$ increases to $15^{\circ}$ and $20^{\circ}$, the reconstruction error ramps up over $2 \mathrm{~dB}$.

The effects of the phase noise error can also be observed through the electric field data misfit $\epsilon_{\mathrm{M}}$ over $\Sigma_{\mathrm{M}}$ [39]-[41]:

$$
\epsilon_{\mathrm{M}}=\sqrt{\frac{\int_{\Sigma_{\mathrm{M}}}\left|\boldsymbol{E}_{\mathrm{n}}^{\mathrm{rec}}(\boldsymbol{r})-\boldsymbol{E}_{\mathrm{n}}^{\operatorname{sim}}(\boldsymbol{r})\right|^{2} \mathrm{~d} s}{\int_{\Sigma_{\mathrm{M}}}\left|\boldsymbol{E}_{\mathrm{n}}^{\operatorname{sim}}(\boldsymbol{r})\right|^{2} \mathrm{~d} s}}, \quad \boldsymbol{r} \in \Sigma_{\mathrm{M}},
$$

where $\boldsymbol{E}_{\mathrm{n}}^{\mathrm{rec}}$ is the electric field reconstructed from $\boldsymbol{E}_{\mathrm{n}}^{\mathrm{sim}}$. In Table II, $\epsilon_{\mathrm{M}}$ is within 0.2 when $\sigma \leq 10^{\circ}$, while it rises rapidly from about 0.2 to about 0.6 in the range $10^{\circ} \leq \sigma \leq 15^{\circ}$ for both $28 \mathrm{GHz}$ and $38 \mathrm{GHz}$.

\section{Position of Device under Test}

During the IF measurements, the connector for each antenna under test is kept at the origin of the spherical coordinate system, even though it is unclear whether this position is the best for the EQC method. Furthermore, for commercial products, the positions of internal integrated antennas may be unknown when measurements are conducted by a third party. Thus, a study of the reconstruction error related to the DUT position is needed.

Here, for an antenna under test the reconstruction errors are computed by placing the DUT at two positions:

1) the center of the connector for an antenna under test is positioned at the origin, referred to as Position A;

2) the geometric center of the DUT is positioned at the origin, referred to as Position B, which is a natural choice when the position of the integrated antenna inside the DUT is unknown.

Fig. 13 shows the origin $O_{\mathrm{A}}$ of Position A and the origin $O_{\mathrm{B}}$ of Position B for Ant 4. The distance between them is about $39 \mathrm{~mm}$, approximately $3.6 \lambda$ at $28 \mathrm{GHz}$ and $4.9 \lambda$ at $38 \mathrm{GHz}$. For Ant 1 -Ant 3, such a distance is even larger. Since such a distance is small compared to the radius of $\Sigma_{\mathrm{M}}$, placing at these two positions mainly results in meaningful difference in the phase distribution of the IF data but not in the amplitude distribution. The $\left|\boldsymbol{J}_{\text {eq }}\right|$ distributions on $\Sigma_{\mathrm{R}}$ for Position A and Position B of Ant 3 at $28 \mathrm{GHz}$ can be found in Fig. 14. Fig. 15 shows the corresponding reconstruction errors. They have the 


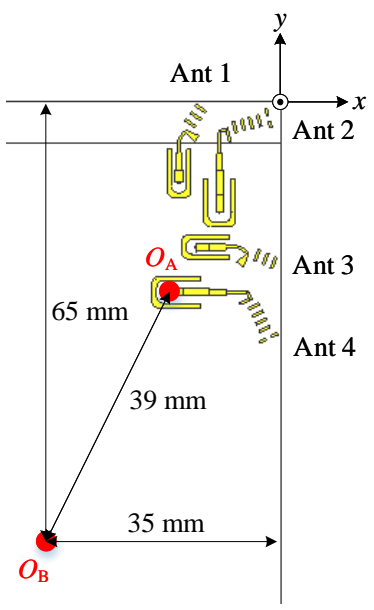

Fig. 13. For Ant 4 under test, the origin of the spherical coordinate system can be set at Position A $\left(O_{\mathrm{A}}\right)$ or Position $\mathrm{B}\left(O_{\mathrm{B}}\right)$.

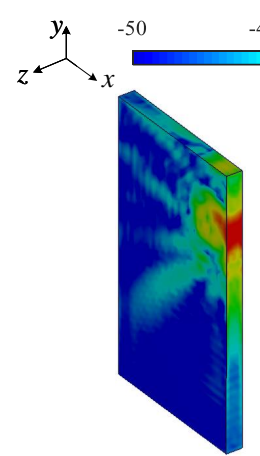

(a)

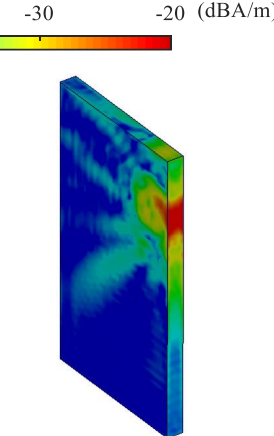

(b)
Fig. 14. $\left|\boldsymbol{J}_{\text {eq }}\right|$ distributions reconstructed from the simulated IF data of Ant 3 at $28 \mathrm{GHz}$ when the origin is set at (a) Position A and (b) Position B.

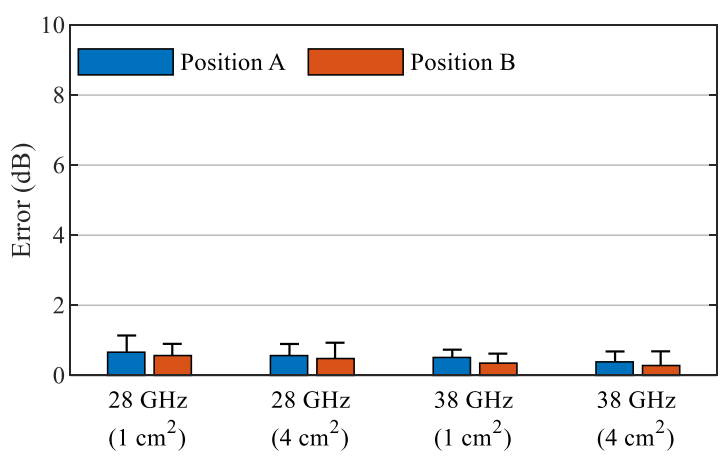

Fig. 15. The mean and maximum values of the reconstruction errors for the $1 \mathrm{~cm}^{2}$ and $4 \mathrm{~cm}^{2}$ psaPD at $28 \mathrm{GHz}$ and the $1 \mathrm{~cm}^{2}$ and $4 \mathrm{~cm}^{2}$ psaPD at $38 \mathrm{GHz}$ reconstructed from the simulated IF data when the origin is set at different positions. Each bar contains 124 samples from different combinations of antenna (Ant 1-4) and evaluation distance (from $d=5 \mathrm{~mm}$ to $d=35 \mathrm{~mm}$ with $1 \mathrm{~mm}$ step).

similar mean value and the similar maximum value of the reconstruction errors, suggesting that the EQC method is not sensitive to the positioning offset.

\section{Discussion}

In terms of measurement time, the present EQC method associated with the spherical measurement system StarLab $50 \mathrm{GHz}$ with $\Delta \alpha=2^{\circ}$ would only take about 10 minutes for one antenna over a wide frequency range. Using the data collected within the measurement, the electric and magnetic fields can be computed in the entire space outside the reconstructed surface at once. For example, the results shown in Fig. 7 and Fig. 8 are based on the data measured in total four tests (one test for every antenna). In contrast, the planar scanning is quite slow, and usually takes one to a few hours for one antenna at a single frequency, depending on the size of the scan area and the frequency, and multiple measurements may be needed to capture the maximum PD for the DUT like a mobile handset.

The present EQC method allows to reconstruct the EQCs on an arbitrary shape and compute fields conformal to the surfaces. On the contrary, for previous methods based on cylindrical or spherical wave expansion techniques, the evaluation surface needs to be cylindrical or spherical [34], and the fields between the minimum cylindrical or spherical evaluation surface and the DUT cannot be evaluated.

For the present EQC method at both $28 \mathrm{GHz}$ and $38 \mathrm{GHz}$, the largest reconstruction errors for both the point PD evaluated at $d=5 \mathrm{~mm}$ and the $p s a P D$ evaluated within the range of $5 \mathrm{~mm} \leq d \leq 35 \mathrm{~mm}$ are no larger than $1.1 \mathrm{~dB}$. In contrast, the highest reconstruction error reported in [20] using the approximate method is about $50.2 \%(1.8 \mathrm{~dB})$ for $p s a P D$, and the highest reconstruction error reported in [27] using the combination of source reconstruction and calibration technique is about $67 \%(2.2 \mathrm{~dB})$ for point PD. The reconstruction error of psaPD using the pseudo-vector electric field measurement technique reported in [22] is less than $0.5 \mathrm{~dB}$ when $d \geq 0.2 \lambda$, and the reconstruction error of psaPD using the plane wave expansion technique reported in [25] and [26] is smaller than $0.4 \mathrm{~dB}$ when $d \geq 0.2 \lambda$. As it is hard to compare the performance for different PD measurement methodologies under different measurement conditions (e.g., different DUT, measurement equipment, and post-processing), the accuracy of the PD assessment using the present EQC method should be considered acceptable in general. In the future, benchmarking and system validation are needed for different PD measurement systems using the DUT with well quantified numerical solutions. Considering the huge benefits brought by the present PD assessment methodology, the number of test configurations required for the full PD assessment can be largely reduced. Thus, the proposed methodology is particularly suitable for fast PD assessment.

This paper mainly focuses on the applicability and requirements of the EQC method for the PD assessment, while in the future, a more comprehensive uncertainty analysis is required considering the sources of uncertainty from the measurement equipment. Also, at even higher frequencies above $38 \mathrm{GHz}$, while at the higher frequency, requirements on the measurement system become inevitably stringent because of the smaller $(\Delta \alpha)_{\max }$, e.g., at $100 \mathrm{GHz}$ for the size of our DUT, $(\Delta \alpha)_{\max }=1.1^{\circ}$. 


\section{CONCLUSION}

In this work, a fast PD assessment methodology using the EQC method for $5 \mathrm{G}$ mmWave mobile handsets has been developed and evaluated. The reconstruction error of the psaPD averaged over $1 \mathrm{~cm}^{2}$ and $4 \mathrm{~cm}^{2}$ based on simulation results is less than $1.1 \mathrm{~dB}$ within $5 \mathrm{~mm} \leq d \leq 35 \mathrm{~mm}$ at $28 \mathrm{GHz}$ and $38 \mathrm{GHz}$, which is generally acceptable compared with other PD assessment methodologies. The antennas and directions with highest PD levels have been successfully identified at different frequencies in greatly reduced total measurement time compared to the conventional methods. Three influencing factors related to the measurement accuracy, i.e., the angular resolution, the phase error, and the DUT position in the IF measurements, have also been analyzed. For our DUT, the angular resolution should be at least $\Delta \alpha=4^{\circ}$ at $28 \mathrm{GHz}$ and $\Delta \alpha=2^{\circ}$ at $38 \mathrm{GHz}$, and the phase error should be within the level of $\sigma=10^{\circ}$. While the reconstruction performance of the EQC method is not sensitive to the DUT position. The analyses and results show that the present EQC method with the multiprobe spherical measurement system can be a good candidate for the purpose of fast $\mathrm{PD}$ assessment of $5 \mathrm{G}$ mmWave mobile handsets.

\section{REFERENCES}

[1] 5G; NR; User Equipment (UE) radio transmission and reception; Part 2: Range 2 Standalone, document TS 38.101-2, 3GPP , Rev. 15.3.0, Oct. 2018.

[2] Draft Guidelines for Limiting Exposure to Time-Varying Electric, Magnetic and Electromagnetic Fields (100 kHz to $300 \mathrm{GHz})$, document, ICNIRP, Jul. 2018.

[3] IEEE Standard for Safety Levels with Respect to Human Exposure to Electric, Magnetic, and Electromagnetic Fields, $0 \mathrm{~Hz}$ to $300 \mathrm{GHz}$, IEEE Std. C95.1-2019, Feb. 2019.

[4] RF Exposure: Order/NPRM Issues, Technical Analysis Branch, Office of Engineering and Technology, FCC, Oct. 2018. [Online]. Available: https://transition.fcc.gov/oet/ea/presentations/files/ oct18/5.1-TCB-RFExposure-OrderNPRM-Issues-MD.PDF

[5] Resolution of Notice of Inquiry, Second Report and Order, Notice of Proposed Rulemaking, and Memorandum Opinion and Order, FCC 19126, Dec. 2019.

[6] B. Xu, K. Zhao, Z. Ying, D. Sjöberg, W. He, and S. He, "Analysis of impacts of expected RF EMF exposure restrictions on peak EIRP of 5G user equipment at $28 \mathrm{GHz}$ and $39 \mathrm{GHz}$ bands," IEEE Access, vol. 7, pp. $20996-21005$, Feb. 2019.

[7] K. R. Foster, M. C. Ziskin, and Q. Balzano, "Thermal response of human skin to microwave energy: a critical review," Health Phys., vol. 111, no. 6 , pp. 528-541, 2016

[8] — - "Thermal modeling for the next generation of radiofrequency exposure limits: commentary," Health Phys., vol. 113, no. 1, pp. 4153, 2017.

[9] R. Morimoto, A. Hirata, I. Laakso, M. C. Ziskin, and K. R. Foster, "Time constants for temperature elevation in human models exposed to dipole antennas and beams in the frequency range from 1 to $30 \mathrm{GHz}$," Phys. Med. Biol., vol. 62, no. 5, pp. 1676-1699, Feb. 2017.

[10] Y. Hashimoto, A. Hirata, R. Morimoto, S. Aonuma, I. Laakso, K. Jokela, and K. R. Foster, "On the averaging area for incident power density for human exposure limits at frequencies over $6 \mathrm{GHz}$," Phys. Med. Biol., vol. 62, no. 8, pp. 3124-3138, Mar. 2017.

[11] K. R. Foster and D. Colombi, "Thermal response of tissue to RF exposure from canonical dipoles at frequencies for future mobile communication systems," Electron. Lett., vol. 53, no. 5, pp. 360-362, 2017.

[12] W. He, B. Xu, M. Gustafsson, Z. Ying, and S. He, "RF compliance study of temperature elevation in human head model around $28 \mathrm{GHz}$ for 5G user equipment application: simulation analysis," IEEE Access, vol. 6, pp. 830-838, Feb. 2018

[13] D. Colombi, B. Thors, C. Törnevik, and Q. Balzano, "RF energy absorption by biological tissues in close proximity to millimeter-wave 5G wireless equipment," IEEE Access, vol. 6, pp. 4974-4981, Feb. 2018.
[14] M. C. Ziskin, S. I. Alekseev, K. R. Foster, and Q. Balzano, "Tissue models for RF exposure evaluation at frequencies above $6 \mathrm{GHz}$," Bioelectromagnetics, vol. 39, no. 3, pp. 173-189, Apr. 2018.

[15] D. Funahashi, A. Hirata, S. Kodera, and K. R. Foster, "Area-averaged transmitted power density at skin surface as metric to estimate surface temperature elevation," IEEE Access, vol. 6, pp. 77 665-77 674, 2018.

[16] K. Li, K. Sasaki, S. Watanabe, and H. Shirai, "Relationship between power density and surface temperature elevation for human skin exposure to electromagnetic waves with oblique incidence angle from $6 \mathrm{GHz}$ to 1 THz," Phys. Med. Biol., vol. 64, no. 6, Mar. 2019.

[17] T. Nakae, D. Funahashi, J. Higashiyama, T. Onishi, and A. Hirata, "Skin temperature elevation for incident power densities from dipole arrays at 28 GHz," IEEE Access, vol. 8, pp. 26863-26871, Jan. 2020.

[18] A. Christ, T. Samaras, E. Neufeld, and N. Kuster, "RF-induced temperature increase in a stratified model of the skin for plane-wave exposure at 6-100 GHz," Radiation Protection Dosimetry, Jan. 2020, ncz293. [Online]. Available: https://doi.org/10.1093/rpd/ncz293

[19] "Measurement procedure for the evaluation of power density related to human exposure to radio frequency fields from wireless communication devices operating between $6 \mathrm{GHz}$ and $100 \mathrm{GHz}$," document TR 63170 , IEC, Aug. 2018.

[20] B. Xu, K. Zhao, B. Thors, D. Colombi, O. Lundberg, Z. Ying, and S. He, "Power density measurement at $15 \mathrm{GHz}$ for RF EMF compliance assessments of 5G user equipment," IEEE Trans. Antennas Propag., vol. 65, no. 12, pp. 6584-6595, Dec. 2017.

[21] B. Xu, M. Gustafsson, S. Zhang, K. Zhao, Z. Ying, and S. He, "Radio frequency exposure compliance of multiple antennas for cellular equipment based on semidefinite relaxation," IEEE Trans. Electromagn. Compat., vol. 61, no. 2, pp. 327-336, Apr. 2019.

[22] S. Pfeifer, E. Carrasco, P. Crespo-Valero, E. Neufeld, S. Kühn, T. Samaras, A. Christ, M. H. Capstick, and N. Kuster, "Total field reconstruction in the near field using pseudo-vector E-field measurements," IEEE Trans. Electromagn. Compat., vol. 61, no. 2, pp. 476-486, Apr. 2019.

[23] cDASY6 Module mmWave, Schmid \& Partner Engineering AG. [Online]. Available: https://speag.swiss/products/dasy6/software/ 5g-module-of-cdasy6/

[24] M. Nesterova, S. Nicol, and Y. Nesterova, "Evaluating power density for 5G applications," in IEEE 5G World Forum (5GWF), Jul. 2018, pp. $347-350$.

[25] K. Sasaki, K. Li, J. Chakarothai, T. Iyama, T. Onishi, and S. Watanabe, "Error analysis of a near-field reconstruction technique based on plane wave spectrum expansion for power density assessment above $6 \mathrm{GHz}$," IEEE Access, vol. 7, pp. 11 591-11 598, Feb. 2019.

[26] K. Sasaki, K. W. K. Li, S. Watanabe, J. Higashiyama, and T. Onishi, "Accuracy of incident power density measured using reconstructing algorithm for compliance assessment of devices in near-field at millimeterwave frequencies," 2019 Joint Int. Symp. Electromagn. Compat., Sapporo and Asia-Pacific Int. Symp. Electromagn. Compat., pp. 43-46, Jun. 2019.

[27] J. Lundgren, J. Helander, M. Gustafsson, D. Sjöberg, B. Xu, and D. Colombi, "Near-field measurement and calibration technique for RF EMF exposure assessment of mm-wave 5G devices," IEEE Antennas Propag. Mag., submitted.

[28] (2019, Jul.) FCC Report A3LSMN976V. [Online]. Available: https: //fcc.report/FCC-ID/A3LSMN976V

[29] (2019, May) FCC Report ZNFV450VM. [Online]. Available: https: //fcc.report/FCC-ID/ZNFV450VM

[30] Human exposure to radio frequency fields from hand-held and bodymounted wireless communication devices - Human models, instrumentations, and procedures - Part 2: Procedure to determine the specific absorption rate (SAR) for wireless communication devices used in close proximity to the human body (frequency range of $30 \mathrm{MHz}$ to $6 \mathrm{GHz}$ ), IEC Std. 62 209-2, Ed. 1.0, Mar. 2010.

[31] K. Persson and M. Gustafsson, "Reconstruction of equivalent currents using a near-field data transformation-with radome applications," Prog. Electromagn. Res., vol. 54, pp. 179-198, 2005.

[32] K. Persson, M. Gustafsson, and G. Kristensson, "Reconstruction and visualization of equivalent currents on a radome using an integral representation formulation," Prog. Electromagn. Res. B, vol. 20, pp. 65$90,2010$.

[33] J. L. A. Quijano, L. Scialacqua, J. Zackrisson, L. J. Foged, M. Sabbadini, and G. Vecchi, "Suppression of undesired radiated fields based on equivalent currents reconstruction from measured data," IEEE Antennas Wireless Propag. Lett., vol. 10, pp. 314-317, 2011.

[34] Y. Álvarez, F. Las-Heras, and M. R. Pino, "On the comparison between the spherical wave expansion and the sources reconstruction method," 
IEEE Trans. Antennas Propag., vol. 56, no. 10, pp. 3337-3341, Oct. 2008.

[35] — "Reconstruction of equivalent currents distribution over arbitrary three-dimensional surfaces based on integral equation algorithms," IEEE Trans. Antennas Propag., vol. 55, no. 12, pp. 3460-3468, Dec. 2007.

[36] P. Petre and T. K. Sarkar, "Planar near-field to far-field transformation using an equivalent magnetic current approach," IEEE Trans. Antennas Propag., vol. 40, no. 11, pp. 1348-1356, Nov. 1992.

[37] F. Las-Heras, M. Pino, S. Loredo, Y. Álvarez, and T. Sarkar, "Evaluating near-field radiation patterns of commercial antennas," IEEE Trans. Antennas Propag., vol. 54, no. 8, pp. 2198-2207, Aug. 2006.

[38] B. Xu, Z. Ying, L. Scialacqua, A. Scannavini, L. J. Foged, T. Bolin, K. Zhao, S. He, and M. Gustafsson, "Radiation performance analysis of $28 \mathrm{GHz}$ antennas integrated in $5 \mathrm{G}$ mobile terminal housing," IEEE Access, vol. 6, pp. $48088-48$ 101, Sep. 2018.

[39] J. L. A. Quijano and G. Vecchi, "Improved-accuracy source reconstruction on arbitrary 3-D surfaces," IEEE Antennas Wireless Propag. Lett., vol. 8, pp. 1046-1049, 2009.

[40] _ - "Field and source equivalence in source reconstruction on 3D surfaces," Prog. Electromagn. Res., no. PIER 103, pp. 67-100, 2010.

[41] - "Near- and very near-field accuracy in 3-D source reconstruction," IEEE Antennas Wireless Propag. Lett., vol. 9, pp. 634-637, 2010.

[42] StarLab $50 \mathrm{GHz}$ OTA measurement system, MVG. [Online]. Available: https://www.mvg-world.com/en/products/antenna-measurement/ multi-probe-systems/starlab-50-ghz

[43] C. D. Paola, S. Zhang, K. Zhao, Z. Ying, T. Bolin, and G. F. Pedersen, "Wideband beam-switchable $28 \mathrm{GHz}$ quasi-Yagi array for mobile devices," IEEE Trans. Antennas Propag., vol. 67, no. 11, pp. 6870-6882, Nov. 2019.

[44] SV Microwave. [Online]. Available: https://www.svmicrowave.com/ products/search/rf_series/SMPS

[45] Mivrowave Vision Group. INSIGHT. [Online]. Available: https://www.mvg-world.com/en/products/field_product_family/ antenna-measurement-2/insight

[46] T. F. Eibert and C. H. Schmidt, "Multilevel fast multipole accelerated inverse equivalent current method employing Rao-Wilton-Glisson discretization of electric and magnetic surface currents," IEEE Trans. Antennas Propag., vol. 57, no. 4, pp. 1178-1185, Apr. 2009.

[47] CST Studio Suite. [Online] Available: https: //www.3ds.com/products-services/simulia/products/cst-studio-suite/ ?utm_source $=$ cst.com\&utm_medium $=301 \& u t m \_c a m p a i g n=c s t$

[48] E-Field mm-Wave Probe for General Near-Field Measurements, Schmid \& Partner Engineering AG. [Online]. Available: https: //speag.swiss/products/dasy6/probes/new-eummwvx-vector-e-probe/

[49] Product Standard to Demonstrate the Compliance of Wireless Communication Devices With the Basic Restrictions and Exposure Limit Values Related to Human Exposure to Electromagnetic Fields in the Frequency Range From $30 \mathrm{MHz}$ to $6 \mathrm{GHz}$ : Hand-Held and Body Mounted Devices in Close Proximity to the Human Body, CENELEC Std. EN 50 566, Oct. 2017.

[50] Determining the peak spatial-average specific absorption rate $(S A R)$ in the human body from wireless communications devices, $30 \mathrm{MHz}$ to 6 $\mathrm{GHz}$ - Part 1: General requirements for using the finite-difference timedomain (FDTD) method for SAR calculation, IEC/IEEE Std. 62 704-1, Ed. 1.0, Oct. 2017.

[51] O. M. Bucci and G. Franceschetti, "On the spatial bandwidth of scattered fields," IEEE Trans. Antennas Propag., vol. 35, no. 12, pp. 1445-1455, Dec. 1987.

[52] - "On the degrees of freedom of scattered fields," IEEE Trans. Antennas Propag., vol. 37, no. 7, pp. 918-926, Jul. 1989.

[53] O. M. Bucci, C. Gennarelli, and C. Savarese, "Representation of electromagnetic fields over arbitrary surfaces by a finite and nonredundant number of samples," IEEE Trans. Antennas Propag., vol. 46, no. 3, pp. 351-359, Mar. 1998.

[54] J. E. Hansen, Ed., Spherical Near-Field Antenna Measurements. Peter Peregrinus Ltd., on behalf of IET, London, United Kingdom, 1988. 